\title{
Los problemas en la metodología tradicional de la investigación arqueoastronómica en sitios arqueológicos de difícil acceso: aplicación al caso del Valle de Jesús de Otoro
}

Cristina Margarita Argueta, Javier Mejuto Gonzalez

\section{Resumen}

Muchos de los sitios arqueológicos registrados en Honduras se encuentran en propiedades privadas o en estado de mal de conservación lo que los hace poco accesibles para los estudios arqueoastronómicos realizados con la metodología no invasiva y tradicional de campo, a través del uso de técnicas astrogeodésicas clásicas. El valle de Jesús de Otoro, rico en vestigios de asentamientos lencas, presenta una serie de problemas para su investigación -en particular para su estudio arqueoastronómico- que van desde la ubicación de sitios en propiedad privada hasta estructuras invadidas por la abundante vegetación. Otro problema importante está asociado a un avanzado estado de deterioro por lo que la toma de datos para su estudio se torna en ocasiones imposible.

Este trabajo propone el uso de las modernas técnicas geodésicas y de detección remota como alternativa a las tradicionales técnicas usadas en los estudios arqueoastronómicos, basándose en el caso de los datos recolectados en el Valle de Jesús de Otoro a través de las técnicas usadas tradicionalmente.

Palabras claves: Arqueoastronomía, SIG, Metodología, Valle de Jesús de Otoro

\section{Abstract}

Many of honduran archaeological sites are located in private properties or badly preserved, this situation make these sites difficult to study from the archaeoastronomical point of view by classical astrogeodesical methods. Otoro Valley is a well 
known rich area in lenca archaeological sites, despite of that it presents several problems for research purposes, particularly for Archaeoastronomy. Between them we have mainly property or entry issues, bad conservation status and invaded or partially invisible structures because of the tropical vegetation. In this work the use of modern geodesical techniques is proposed for archaeoastronomical studies in this type of context as alternative to classical techniques using Jesus de Otoro Valley as a case study.

Keywords: Archaeoastronomy, GIS, Methodology, Jesus de Otoro Valley

Cristina Margarita Argueta, Javier Mejuto Gonzalez, Departamento de Arqueoastronomía y Astronomía Cultural, Universidad Nacional Autónoma de Honduras 


\section{Introducción}

La selección de una correcta metodología es vital para los estudios de campo, especialmente en los contextos culturales arqueológicos ya que en muchas ocasiones no podemos repetir las mediciones por la desaparición del patrimonio, en muchas ocasiones debido a una mala gestión patrimonial. De forma continuada, y cada vez con mayor frecuencia, se han venido utilizando en campo -como en el caso de estudio que aparece en estas líneas- instrumentos y técnicas que poseen una probada precisión en campo como son los sistemas de posicionamiento global por satélite (GNSS). Sin embargo, como todo sistema tiene sus limitaciones técnicas que en un contexto geográfico tropical pueden invalidar nuestras conclusiones y en el peor de los casos incluso imposibilitar su uso por completo.

El valle Jesús de Otoro fue conocido como Jurla, y posteriormente como San Juan de Quelala para finalmente tomar el nombre de Jesús de Otoro con una extensión territorial de $406.6 \mathrm{Km}^{2}$. El valle está ubicado en la zona central de Honduras, limita al norte con los municipios de San José de Comayagua y San Pedro Zacapa; al sur con los municipios de Masaguara e Intibucá; al este con el municipio de Siguatepeque y al oeste, con los municipios de San Isidro e Intibucá. Se trata de un zona de gran riqueza arqueológica y patrimonial (Cruz, 2004) pero en la que la mayoría de los sitios arqueológicos se encuentran en estado grave de deterioro, invadidos principalmente por la agricultura, asentamientos humanos y las arboledas (ver figura 1). Es por ello que se han venido concentrando esfuerzos para registrar estos sitios arqueológicos en vista de un proyecto global que aúna esfuerzos para su estudio y conservación. El análisis que aparece en estas líneas tiene como finalidad realizar el registro de la mejor manera posible para futuros trabajos ya proyectados en la zona. En este caso nos referiremos a los sitios de La Canoa, San Marcos, Aguas Blancas, Guayamán y Mixcure que se sitúan entre la Quebrada de Otoro por el sur y el Río Siruna por el norte (Véliz et al.,2014).

\section{Metodología}

Los sistemas de posicionamiento global por satélite han sido frecuentemente usados en contextos arqueológicos, en especial tras la aparición de nuevas constelaciones satelitales como GLONASS y GALILEO que han mejorado la cobertura de esta tecnología. En arqueología su uso prioritario ha sido la georreferenciación de hallazgos, tanto para la prospección arqueológica superficial como para la excavación metodológica. Se necesita la mayor exactitud posible para determinar la localización de los yacimientos sobre el terreno para conocer su distribución espacial como para su estudio posterior volviendo a los mismos o en gabinete. Para su uso debemos tener en consideración las diferentes fuentes de error que están relacionados con el propio satélite, su señal y el receptor. Podemos ver los errores y valores típicos de los mismos en la tabla 1, pero en este caso nos centraremos en los errores que podemos sufrir por el uso de receptores autónomos en toma de datos. 


\begin{tabular}{l|rr}
\hline Reloj del satélite & $1.5 \mathrm{~m}$ & $0 \mathrm{~m}$ \\
Error orbital & $2.5 \mathrm{~m}$ & $0 \mathrm{~m}$ \\
Ionosfera & $5 \mathrm{~m}$ & $0.4 \mathrm{~m}$ \\
Troposfera & $0.5 \mathrm{~m}$ & $0.2 \mathrm{~m}$ \\
Ruido del receptor & $0.3 \mathrm{~m}$ & $0.3 \mathrm{~m}$ \\
Multipath & $0.6 \mathrm{~m}$ & $0.6 \mathrm{~m}$ \\
Disponibilidad selectiva & $30 \mathrm{~m}$ & $0 \mathrm{~m}$ \\
\end{tabular}

Tabla 1. Fuentes típicas de error en sistemas GNSS y sus valores típicos

El hecho de que unas coordenadas individuales sean inexactas puede reducirse de forma considerable con los métodos diferenciales. En ellos intervienen dos receptores simultáneamente, uno de ellos se coloca en un punto que conozcamos sus coordenadas con gran exactitud -un vértice geodésico suele ser lo más utilizado- conocida como estación de referencia. El otro receptor se coloca en el punto a medir, ya sea este variable si tenemos que medir varios puntos o fijo si tenemos que medir solamente un punto nuevo cuyas coordenadas queremos encontrar. Los receptores funcionan simultáneamente pero los datos de la estación de referencia pueden utilizarse para conocer qué error se está cometiendo en las coordenadas del punto que se está midiendo. Estas correcciones pueden realizarse en tiempo real, mediante la conexión entre ambos receptores (esto es posible típicamente hasta una distancia máxima de unos 20 kilómetros de separación) 0 posteriormente con los datos de ambas estaciones.

En análisis realizado en el Valle Jesús de Otoro refleja dos problemas típicos del uso de este tipo de técnicas en campo en trabajos arqueológicos y arqueoastronómicos. La falta de correcciones GNSS que aumente la precisión de las medidas y la falta de estudios de altura de horizonte. Según el estudio realizado por Véliz y colaboradores el procedimiento de trabajo consistió en tomar puntos en cada esquina de la estructura principal de los sitios arqueológicos estudiados (Véliz et al., 2014), con un receptor autónomo durante medidas de 60 segundos para -a través de las coordenadas geográficas- conseguir el acimut de los líneas de pared para cada estructura. El método puede consultarse, por ejemplo, en Wolf (Wolf y Ghilani, 2002, 582-ss) que utiliza la desarrollada por Vicenty (Vincenty, 1975). 
Esta metodología, que en ocasiones puede ser suficiente para ubicar hallazgos de piezas o delimitar sitios arqueológicos complejos, puede no serlo en contextos como el que nos encontramos en el Valle de Otoro. De hecho, en el trabajo en este valle confluyen todas las circunstancias para que el trabajo con sistemas GNSS sea realmente complicado.

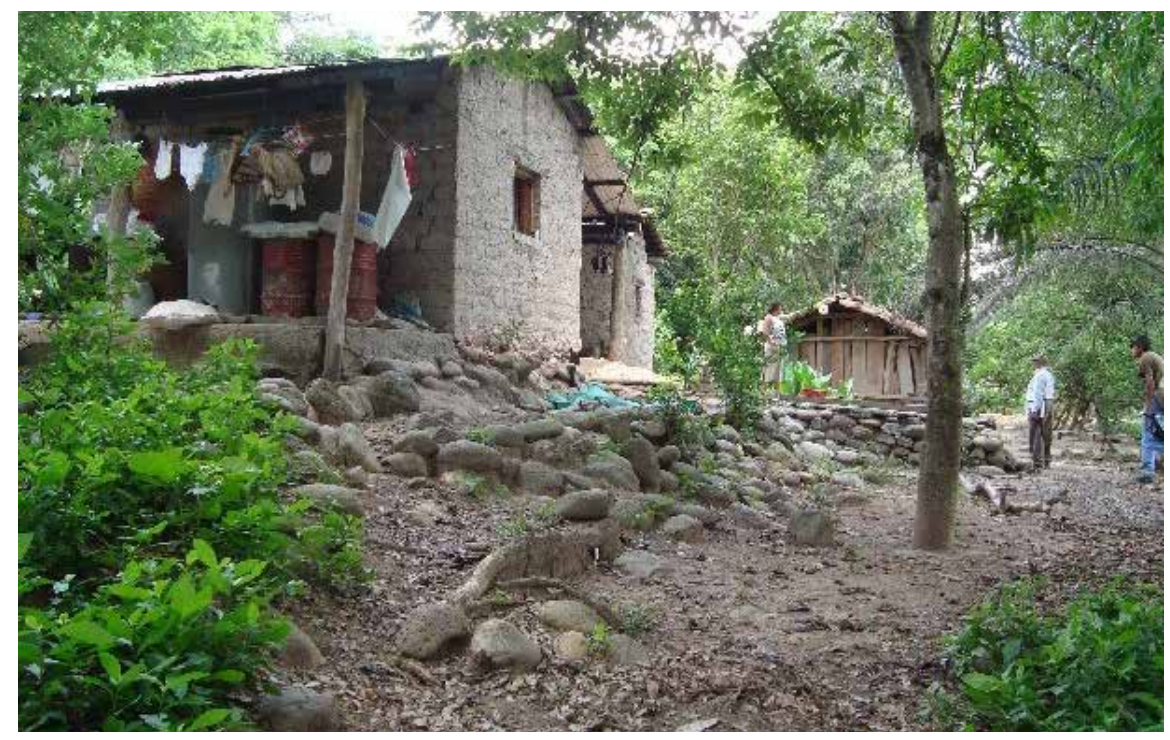

Figura 1. Situación de algunas estructuras estudiadas en el valle de Otoro

La cantidad de masa vegetal que rodea los sitios hace que errores de multipath y falta de señal aumentan de forma considerable la incertidumbre en la precisión de las coordenadas a medir. Por otro lado, el estado de conservación y la invasión de otras estructuras como se ve en la figura 1, dificultan conocer con exactitud dónde se encuentran las esquinas medidas por los investigadores. Ya por último, la misma densidad vegetal hace imposible realizar estudios topoastronómicos y simulaciones del cielo en la época de estudio.

Algunos cambios en la metodología de trabajo como la inclusión de técnicas GNSS diferenciales o la medición de varios puntos en las líneas de pared de las estructuras en estudios en lugar de la medición de esquinas solamente parece que pueden mejorar sustancialmente la precisión de los resultados de ángulo acimut. De no hacerlo, podemos estar concluyendo resultados erróneos a partir de los datos obtenidos en el estudio (ver tabla 2). 


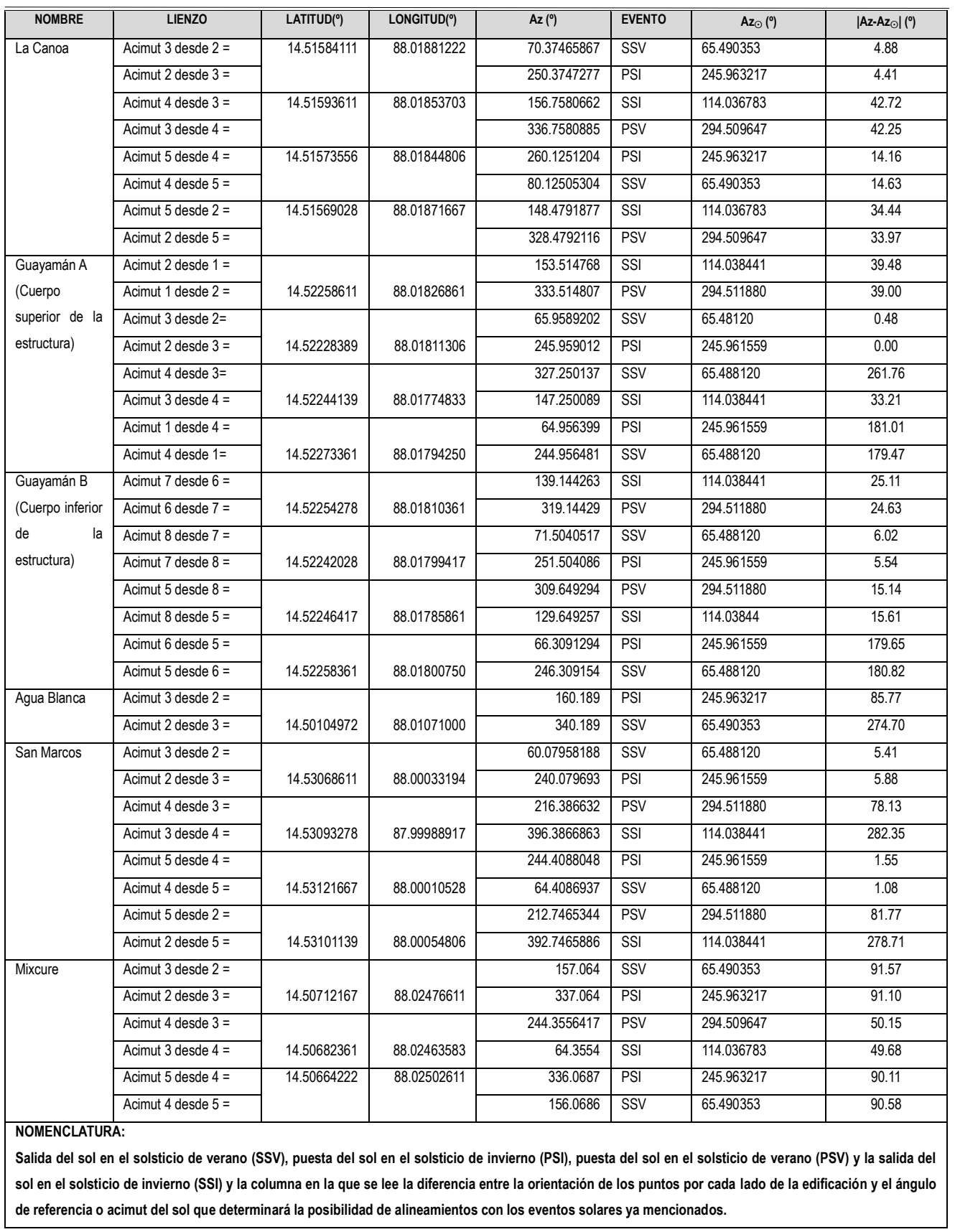

Tabla 2. Acimutes de las líneas de pared de las estructuras estudiadas y sus diferencias en valor absoluto. Tomado de Véliz et al. (2014) 
Podemos ver en la tabla 2 algunas alineaciones con desviaciones menores de un grado que bien pueden ser mucho mayores debido a los errores producidos en el receptor GNSS autónomo las circunstancias locales de medida. Del mismo modo, también existen otros valores de desviaciones que a pesar de ser aparentemente altos -hasta varios grados- pueden esconder alineaciones debidos a que se han sobreestimado estos valores de acimut. Aún mayor es el problema de la falta de estudios que tengan en consideración la altura del horizonte, en extremo importante para cálculos y visibilidad local de eventos astronómicos además de marcadores conspicuos en el horizonte local observable que permitan marcar momentos en dichos eventos astronómicos.

Por lo tanto, debido a las situaciones y complicaciones a la hora de tomar los datos debemos ser escépticos antes los resultados -tanto favorables como desfavorables- de las orientaciones de las estructuras estudiadas en el valle de Otoro.

\section{Discusión}

Según el análisis de los datos para el cálculo de los alineamientos, el resultado es positivo para los sitios que visiblemente están en condiciones favorables de conservación como la el cuerpo superior de la estructura registrada en Guayamán, sin embargo para el resto de sitios que se encuentran en malas condiciones de conservación y cuya toma de datos son de supuestos vértices, no existe coincidencia de alineamientos.

Como se ha comentado, estos resultados pueden no corresponder a la realidad debido a los condicionantes en los que se han tomado los datos. Sería recomendable repetir las mediciones con técnicas avanzadas utilizando técnicas combinadas astrogeodésicas y geodésicas con sistemas de posicionamiento global por satélite con las que podemos obtener precisiones que llegan al milímetro o al segundo de arco dependiendo de la técnica empleada (Mejuto y Rodríguez, 2010). Si seguimos interesados en utilizar este tipo de técnicas para el estudio, debemos hacer un análisis de métodos e instrumentación correspondiente para conseguir el resultado requerido con la mayor eficacia y eficiencia. Para ello podemos utilizar una gráfica de decisión como la que aparece en la figura 2, en la que se tiene en consideración variables como precisión de medida, el tiempo que se invierte en la toma y análisis de los datos, el coste del equipamiento para realizar la técnica y la complejidad, estado de conservación y número de restos arqueológicos o estructuras a estudiar.

Por otro lado, dados los problemas con la vegetación -típicos de los trabajos en estas latitudes- sería ideal para futuros trabajos cambiar la metodología utilizada hacia las infraestructuras de datos espaciales (IDE) o métodos de percepción remota como el Light Detection and Ranging (LIDAR), siendo las IDEs una técnica mucho más accesible por su coste. 
Habitualmente se han venido utilizando las IDEs y, en particular, las Sistemas de Información Geográfica como un catálogo virtual de yacimientos arqueológicos sin utilizar las capacidades de geoprocesado propias de estas técnicas. En particular son de especial interés los cálculos de cuencas de visibilidad, de perfiles de horizonte,...

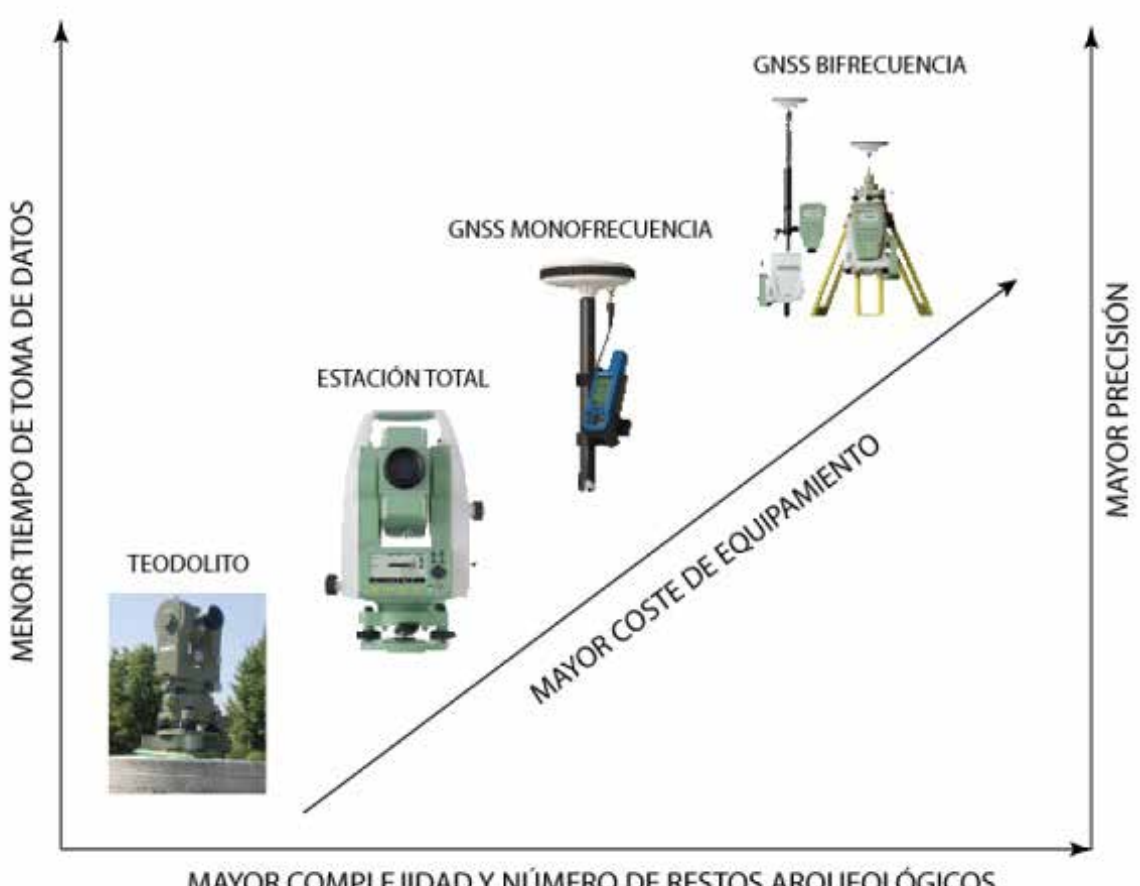

Figura 2. Gráfica de decisión para métodos geodésicos en Arqueoastronomía (Mejuto, 20)

Un ejemplo de la utilidad de los IDEs en Arqueoastronomía pueden verse en Mejuto, en el que se utilizan los Modelos Digitales de Terreno (MDT) como herramienta para el análisis y caracterización de perfiles de horizontes con finalidad astronómica. Se trata por lo tanto, de añadir una capa más a las tradicionales capas de información en los Sistemas de Información Geográfica; es decir, una capa de información astronómica que nos proporcione información astronómica georreferenciada de utilidad arqueoastronómica. 


\section{Conclusión}

La investigación arqueoastronómica en edificios arqueológicos con un alto grado de deterioro o complejidad requiere de un análisis técnico y científico que tenga en consideración las características propias de ser una disciplina que debe considerar aspectos culturales como astronómicos. Por ejemplo, debemos tener en cuenta las consideraciones que cualquier arqueólogo tendría para registrar su yacimiento, junto con las propias de la astronomía que nos permita demostrar el uso de la observación y utilización de eventos astronómicos dentro de los aspectos culturales. Es por ello que un correcto análisis de cada uno de los sitios arqueológicos en estudios -en particular para la investigación a realizar en el valle de Otoro- es de vital importancia para un trabajo eficiente y eficaz que maximice los recursos tan escasos en los proyectos patrimoniales.

Por lo tanto, se propone la utilización de técnicas apegadas a las nuevas tecnologías que posibiliten la reducción al máximo de los efectos negativos de la vegetación para el análisis de estructuras, así como métodos de registro digitales que reduzcan al máximo el factor humano para la interpretación de elementos de estructuras deterioradas, tales como esquinas de edificios. En el caso del valle de Otoro, todo la información debe permitir crear una IDE que reúna tanto la información geográfica como astronómica para abarcar la totalidad geográfica que conforma el propio valle.

\section{Bibliografía}

- Cruz, O. N. (2004). Patrón de asentamiento prehispánico en el Valle de Jesús de Otoro. Instituto Hondureño de Antropología e Historia, Tegucigalpa.

- Mejuto, J.; Gómez Castaño, J. y Rodríguez-Caderot, G. (2012). Archaeology, New Approaches in Theory and Techniques. capitulo GIS Techniques in Archaeology: An Archaeoastronomical Approach, pp. 117-132. Intech.

- Mejuto, J. y Rodríguez, C. (2010). La metodología en los estudios arqueoastronómicos. Seminario de Arqueología y Etnología Turolense.

- Véliz, V., Rodríguez Carías, C. I., y Argueta Canizales, C. M. (2014). Ubicación de sitios arqueológicos a través de nuevas tecnologías de la Información GEográfica, Valle de Otoro, Honduras. Ciencia y Tecnología, 14:42-61.

- Wolf, Paul R. y Ghilani, Charles D. (2002). Elementary Surveying: An Introduction to Geomatics. Prentice Hall. 